\title{
Efficacy and Cost-Effectiveness of Anti-VEGF for Treating Diabetic Retinopathy in the Indian Population
}

\author{
Subhasish Pramanik' \\ Lakshmi Kanta Mondal (iD) ${ }^{2}$ \\ Suman Kalyan Paine ${ }^{3}$ \\ Sneha Jain ${ }^{2}$ \\ Subhankar Chowdhury' \\ Upasana Ganguly (D) ${ }^{4}$ \\ Sayantan Ghosh ${ }^{2}$ \\ Chiranjit Bose' \\ Koena Bhattacharjee ${ }^{2}$ \\ Gautam Bhaduri ${ }^{5}$ \\ 'Institute of Post Graduate Medical \\ Education and Research (IPGMER), \\ Kolkata, 700020, India; ${ }^{2}$ Regional Institute \\ of Ophthalmology (RIO), Medical College \\ and Hospital, Kolkata, 700073, India; \\ ${ }^{3}$ National Institute of Biomedical \\ Genomics (NIBMG), Kalyani, West \\ Bengal, India; ${ }^{4}$ Maharishi Markandeshwar \\ Institute of Medical Sciences and \\ Research, Mullana, Haryana, India; ${ }^{5}$ MGM \\ Medical College, Kishanganj, Bihar, India
}

Purpose: In the present study, we aimed to evaluate the efficacy, safety, and costeffectiveness of the anti-vascular endothelial growth factor (anti-VEGF), namely ranibizumab (RBZ) or bevacizumab (BVZ), after either focal or grid or scatter laser photocoagulation, for the treatment of diabetic macular edema (DME) in the Indian population. Methods: Retrospective data were collected in the Regional Institute of Ophthalmology, Kolkata, India between January 2018 and June 2019. Seventy-seven eyes received 3 consecutive monthly intravitreal injections of RBZ $(0.5 \mathrm{mg})$ and were followed by prompt laser photocoagulation (within 7-10 days after the third injection). Similarly, 51 eyes received 3 consecutive monthly intravitreal injections of BVZ $(1.25 \mathrm{mg})$, an off-label drug, and were followed by prompt laser therapy. Safety assessments of the therapy, as well as surrogate markers of biochemical derangements related to diabetic retinopathy (DR), were also investigated at the end of 12 months.

Results: Seventy-seven subjects who were given a treatment of RBZ+laser therapy showed average $6.87 \pm 5.53$ letters gain in their best-corrected visual acuity (BCVA) score, whereas the ones treated with off-label BVZ+ laser therapy demonstrated improvement in BCVA of an average 6.82 \pm 5.76 letters in "Early Treatment Diabetic Retinopathy Study" (ETDRS) chart. The study also highlights the cost-effectiveness of both RBZ+laser and BVZ+laser therapies for the treatment of DME in DR. The results demonstrated that a subject has to pay 20.951 times more cost (in INR) for RBZ+laser therapy compared to BVZ+laser therapy, to get an almost similar outcome.

Conclusion: BVZ is found to be the more attractive option for treating DME in DR for its cost-friendliness over RBZ in terms of BCVA outcome, as well as the safety perspectives, at least for the economically backward population in developing countries, like India.

Keywords: diabetic retinopathy, diabetic macular edema, ranibizumab, bevacizumab, laser therapy, cost-effectiveness

\section{Introduction}

Promising therapeutic efficacy of the monoclonal antibody against the Vascular Endothelial Growth Factor (VEGF) has led clinicians' vis-à-vis VEGF antagonists, all, to opt to shift the treatment paradigm from painful conventional laser treatment - followed by surgery - to pharmacotherapy in the treatment of many macular diseases in the technologically advanced industrial countries of the world. In other parts of the world, pharmacotherapy is fast replacing the traditional treatment regime of "conventional laser" for a variety of ocular diseases, like age-related macular degeneration (ARMD) and macular edema (ME). ${ }^{1,2}$ In the treatment of diabetic retinopathy (DR), monoclonal antibodies against VEGF, like ranibizumab
Correspondence: Lakshmi Kanta Mondal Regional Institute of Ophthalmology, Medical College, Kolkata, India Tel +9l 9830830216

Email Lakshmi.mondal62@gmail.com 
(RBZ), off-label bevacizumab (BVZ), and aflibercept (soluble decoy receptor) have gained acceptance through a series of successful randomized control trials (RCT). ${ }^{3-6}$ Several genomic studies, as well as those for proteomic cases, replicate the thought that VEGF plays a central role in the pathogenesis of DR through VEGF's interaction with its three receptor tyrosine kinases viz. Flt-1, KDR, and VEGFR3 ${ }^{2,4}$ This knowledge about VEGF's role at the level of DR pathogenesis of the eye invites the therapeutic interventions to target VEGF for treatment of DR. Monoclonal antibody against VEGF, viz. RBZ, BVZ, and aflibercept seem to be the accepted treatment in the eyeclinic after a series of trials. ${ }^{6}$ RBZ is used as the FDAapproved therapeutic for wet ARMD and macular edema following retinal vein occlusion, as well as in diabetic macular edema (DME); but, BVZ is also administered widely as an off-label drug due to its low cost. ${ }^{6}$ India ranks second in the prevalence of diabetes mellitus and the number of people with DM is expected to increase from the current 67 million to 79.4 million by 2030 . In India, the prevalence of any type of DR is $15.4 \%$ of which $6.7 \%$ accounts for sight-threatening retinopathy. ${ }^{7}$ The number of diabetics with DR will increase to 22.4 million in the next decade which will result in a heavy burden on the health-care system because of the high cost of anti-VEGF drugs. ${ }^{8}$ The global perspective highlighted that 93 million people around the world are affected by the vision-threatening DR. ${ }^{7}$ The present scenario seems to point accurately at an alarming time for the globe as diabetes is increasing exponentially with the economic prosperity and upscaling of living standards of the people which indicate that about $8.5 \%$ of the world's population is affected by the disease. ${ }^{7,8}$ Advancement of anti-VEGF therapy, viz. RBZ and BVZ may be used as a strong weapon to fight the occurrence of blindness due to DR, though the cost of treatment remains a significant concern at least for economically impoverished people, particularly in the treatment using the FDA-approved RBZ. Previous randomized controlled clinical trials of anti-VEGF therapy- plus prompt or deferred later revealed that about $50 \%$ of study subjects - with centre-involving DME - improved their vision where they achieved $\geq 10$ letters, and $30 \%$ achieved $\geq 15$ letters on the ETDRS scale which is higher than what is achieved by conventional laser therapy alone, after a median number of injections, ${ }^{8,9}$ injections of RBZ administered. ${ }^{9}$ So, a notable success on one side and significant cost on the other build the crucial horns of the dilemma on how to apply this promising anti-VEGF therapy on a group of majorly poor Indian people, particularly for RBZ, without imposing monetary hardship on them. The present study may provide substantial clinical information on the efficacy of anti-VEGF therapy using RBZ or BVZ plusprompt-focal-or-grid-laser among the individuals having one among many varied forms of vision-threatening DR, as we tried to evaluate the cost-effectiveness, the efficacy, and the safety for intra-vitreal administration of BVZ or RBZ for the treatment of DME involving the center of the macula.

\section{Methods}

Study design: It was a retrospective study on the data collected in Eastern India.

Location of the study: This study was done at the Regional Institute of Ophthalmology, Medical College, Kolkata, India. The period of record collection was from January 2019 to June 2020.

Study population: The study population consisted of 128 patients of $\geq 45$ years of age with type 2 DM (as per the American Diabetes Association and WHO guidelines) having DR and vision impairment due to centre-involving DME.

\section{Diagnosis}

The grading and the severity of DR, with DME, were determined by trained ophthalmologists (LKM and SG) using (i) dilated fundus examination with slit-lamp-bio-microscopy by +90D and 3-mirror-lens, (ii) seven-field-digital-fundusphotography with fluorescence angiography, and (iii) Optical coherence tomography (Spectral-domain OCT). Central Macular Thickness (CMT) was determined through Spectralis OCT (Heidelberg Engineering, Heidelberg, Germany). Volume scan was performed on a $20 \times 20$ degree cube with 49 raster lines, each containing 1064 pixels, separated by $120 \mu$. The high acquisition speed of $40,000 \mathrm{~A}$-scans/ second avoided artifacts from microsaccades and improved image definition. The spectral mapping software generates automated measures of retinal thickness based on analysis of the central and inner 1000, 3000, and $6000 \mu \mathrm{m}$ subfields as defined by the Early Treatment Diabetic Retinopathy Study.

The grading and the severity of retinopathy were based on the criteria of the modified Early-Treatment Diabetic Retinopathy Study (ETDRS). Best Corrected Visual Acuity (BCVA) was measured using the ETDRS chart at a distance of 4 meters, first, at baseline (prior therapy) and, then, at every visit before the injections and the endpoint 
ie, at month 12. The OCT measurement of the central macular thickness (CMT) also followed the same regime.

The main categories of treatment disorders, assessed from the data collected from the repository of individuals at the tertiary center, were as follows: (i) DME type (focal/ diffuse) in moderate and severe non-proliferative diabetic retinopathy (NPDR) and proliferative diabetic retinopathy (PDR), (ii) baseline best-corrected visual acuity (BCVA) status in US Equivalent Notations, and (iii) visual acuity letter score as 20/50 - 20/63 (letter score 80-75), 20/100 20/125 (letter score 65-60), 20/160 - 20/200 (letter score 55-50); given range of central macular thickness (350-407 $\mu \mathrm{m}, 419-558 \mu \mathrm{m}, 624-715 \mu \mathrm{m})$ and laser application type (focal and/or grid and/or scatter). The ischemic variety of DME was excluded from the treatment regime.

Inclusion criteria: 1) Patients having reduced vision due to non-ischemic focal or diffuse clinically significant DME involving the center of macula in at least one eye that was eligible for laser treatment in the opinion of the treating clinician.

2) All diagnosed and well-controlled type 2 DM.

3) Only newly diagnosed cases of DR with clinically significant macular edema involving the center of the macula.

4) Patients having visual acuity between $20 / 50$ and 20/200.

5) Patients did not receive any form of treatment (focal/grid/scatter laser or injection of anti-VEGF agents or intravitreal steroids).

The key exclusion criteria were (1) concomitant conditions in the study eye that could prevent the improvement in visual acuity on the treatment of DME; (2) presence of intraocular inflammation or infection in either eye; (3) uncontrolled glaucoma; (4) previous laser photocoagulation; and (5) history of stroke and uncontrolled hypertension.

The study was approved by the institutional Ethics Committee and informed consents were collected from all patients according to the declaration of Helsinki.

\section{Treatment Modalities}

Seventy-seven eyes received 3 consecutive monthly intravitreal injections of RBZ $0.5 \mathrm{mg}$ and were followed by prompt laser photocoagulation (within 7-10 days after the third injection). Similarly, 51 eyes received 3 consecutive monthly intravitreal injections of $\mathrm{BVZ}$ $1.25 \mathrm{mg}$, an off-label drug, and were followed by prompt laser therapy. Intravitreal anti-VEGF injections were performed aseptically and followed the clinicians' usual routines; both pre- and post-injection topical antibiotics.

Firstly, a modified ETDRS focal/grid laser photocoagulation technique was used in our treatment protocol. We directly treated all microaneurysms in areas of retinal thickening between 500 and $3000 \mu \mathrm{m}$ from the center of the macula. A mild grey-white burn of $50 \mu \mathrm{m}$ spot size and burn duration of 0.1 seconds was applied beneath all microaneurysms. Secondly, in grid treatment, a laser burn was applied to all areas of the retinal thickness not associated with microaneurysms. The area considered for grid treatment was 500-3000 $\mu \mathrm{m}$ superiorly, nasally, and temporally from the center of the macula. No burn was placed within $500 \mu \mathrm{m}$ of the disc. Panretinal photocoagulation followed the Diabetic Retinopathy Study photocoagulation technique as $800-1600$ burns of $500 \mu \mathrm{m}$ spot size, given the burns were of 0.1 -second duration. Lastly, direct treatment of new vessels and microaneurysms, or another lesion-causing macular edema, took place. Retreatment with laser was given following ETDRS guidelines at intervals no earlier than 3 months from the previous treatment if required for recurrence of clinically significant macular edema. Although, all remarkable randomized controlled clinical trials advocated the continuation of monthly antiVEGF injections after the loading phase until stable visual acuity was reached, and superiority of deferred laser in comparison prompt laser treatment, our economic situation with an enormous number of affected population could not provide such treatment to each patient.

\section{Outcome}

The primary efficacy endpoints included mean BCVA letter score change from baseline to month 12 . The secondary efficacy endpoint included a mean reduction in central macular thickness.

The safety assessments of the therapy were done by a 12-month incidence of adverse events, like endophthalmitis, intraocular hemorrhage, retinal detachment, raised intraocular pressure (IOP), and changes in vital signs, like body temperature, blood pressure, myocardial infarction, and cerebrovascular accident. We also measured surrogate markers of detrimental biochemical pathways which are related to pathogenesis and progression of DR, like advanced glycation end product (AGE) formation, lipid peroxidation, and oxidative stress generation at end of the therapy ie, at the end of the month 12 . 


\section{Calculation of the Cost-Effectiveness}

In India, the cost of treatment for an RBZ (NOVARTIS, India, Ltd) injection was Rs/- 17,500/-, and BVZ (GENENTECH, India Pvt Ltd) was RS/- 1648.35. Costeffectiveness was calculated in terms of the increment of the cost of treatment/letter gain (BCVA improvement)/ subject for RBZ or BVZ therapy compared to counter one.

\section{Laboratory Investigations at the End of 12 Months}

Measurement of reactive oxygen species (ROS) from mononuclear cells of each subject: Mononuclear cells from peripheral blood (PBMCs) were obtained from $6 \mathrm{~mL}$ heparinized blood using Histopaque 1077 (Sigma Aldrich, St Louis, MO, USA). This process separates the media density gradient for $40 \mathrm{~min}$ at 2000 r.p.m and $20{ }^{\circ} \mathrm{C}$. Further, PBMCs were subjected to centrifugation at 2000 r.p.m for 10 minutes and washed twice with 1 $\mathrm{X}$ PBS (pH 7.2). Thereafter, $5 \times 10^{5}$ cells were pelleted into two different tubes and resuspended in $1 \mathrm{X}$ Phosphate-buffered saline (PBS) (pH 7.2) for the estimation of ROS. To measure intracellular ROS generation in mononuclear cells, ROS-sensitive cell-permeable dye 2'7' dihydro dichlorofluorescein diacetate, in the presence of ROS, was oxidized to highly fluorescent $2^{\prime} 7^{\prime}$ dichlorofluorescein in the cell. Production of intracellular ROS is directly proportional to the oxidation of 2'7'dihydrodichlorofluorescein diacetate, elevating the fluorescence level as measured by flow cytometry. ${ }^{10}$

Measurement of total advanced glycation end-products (AGEs) from serum: Serum level of AGEs was measured by enzyme-linked immunosorbent assay method by using the Cell Biolabs kit (Cell Biolabs, San Diego, CA). AGE protein adducts present in the sample were probed with an ant-AGE polyclonal antibody, followed by a horseradish peroxidase (HRP) conjugated antibody. The AGE-protein adduct content in the sample was determined by comparison with a standard curve prepared from AGE-bovine serum albumin (BSA) standards ranging from 0.25 to 5 $\mu \mathrm{g} / \mathrm{mL}$. The absorbance of the final color product was read at $450 \mathrm{~nm}$ wavelength using a Bio-Rad multiplate reader (Model 680, Bio-Rad, Laboratories, Hercules, CA) against the reduced BSA standard as the absorbance blank. ${ }^{11}$

Measurement of MDA: Serum MDA was measured by the colorimetric method as described by Satoh $^{12}$ and reported by Pramanik et al. ${ }^{13}$ In this method, $2.5 \mathrm{~mL}$ of $20 \%$ trichloroacetic acid and $1.0 \mathrm{~mL}$ of $0.67 \%$ thiobarbituric acid were added to $0.5 \mathrm{~mL}$ of serum. The mixture was then heated in a boiling water bath for 30 min. The resulting chromogen was extracted with $4.0 \mathrm{~mL}$ of n-Butyl alcohol, and the absorbance of the organic phase was determined spectrophotometrically at $530 \mathrm{~nm}$.

Follow-up: The evaluation of response to the treatment modality by measuring BCVA score and CMT during every visit of treatment-naïve patients with DME treated by intravitreal injections of ranibizumab (RBZ) and bevacizumab (BVZ) with prompt laser therapy. Every subject was asked to attend the clinic on the first post-operative day and at months 1, 2, 3, 6 at the end of the month, 12 and were followed-up meticulously. For intravitreal antiVEGF, specially BVZ, which is not approved by the FDA, the dreaded complication of endophthalmitis was explained in detail, and patients were instructed to adhere to the follow-up regimen or report at the first sign of worsening vision, pain, and redness. Other complications like mild ocular pain, anterior segment reaction, and an increase in intraocular pressure were monitored carefully. Slit-lamp examination for lens status was done to rule out injury to the lens by a $30 \mathrm{G}$ needle. All patients were examined by indirect ophthalmoscopy with a $+20 \mathrm{D}$ lens to rule out iatrogenic break and retinal detachment.

The patients were fully informed about small floaters, color vision alteration, visual field defects, and night vision problems after laser photocoagulation.

\section{Statistical Analyses}

Data were presented as mean \pm standard deviation (SD). Normality for the data was evaluated by "Shapiro-Wilk Test". Normally distributed variables of two groups were compared by unpaired Student's $t$-test. Not-normally distributed variables of two groups were compared by MannWhitney $U$-test. The distribution of categorical variables in two groups was presented as percentage (\%) and compared by the Chi-Square test. A value of $\mathrm{p}<0.05$ was considered statistically significant. The statistical analyses were performed using statistical software Graph pad prism (Version 5, 2007, San Diego, CA, USA).

\section{Results}

Different study groups enrolled in the present study showed no statistically significant differences for age, duration of diabetes, glycated hemoglobin level (HbAlc $\%$ ), distribution of gender, and subjects with different types of DR (Table 1). 
Table I Baseline Demographic and Disease Characteristics

\begin{tabular}{|c|c|c|c|c|}
\hline \multicolumn{2}{|l|}{ Variables } & \multirow{2}{*}{$\begin{array}{c}\text { RBZ + Laser } \mathbf{N}=\mathbf{7 7} \\
55.2 \pm 9.20\end{array}$} & \multirow{2}{*}{$\frac{\text { BVZ +Laser } \mathbf{N}=\mathbf{5} \text { I }}{560.3 \pm 8.15}$} & \multirow{2}{*}{$\frac{p \text {-value }}{0.489}$} \\
\hline Age [years (Mean \pm SD)] & & & & \\
\hline \multirow[t]{2}{*}{ Gender } & Male & $42(54.54 \%)$ & $26(50.98 \%)$ & \multirow[t]{2}{*}{0.692} \\
\hline & Female & $35(45.45 \%)$ & 25 (49.01\%) & \\
\hline Duration of DM [years (Mean \pm SD)] & & $\mid 1.92 \pm 4.31$ & $13.02 \pm 5.06$ & 0.191 \\
\hline HbAlc (\%) & & $7.56 \pm 3.2$ & $7.42 \pm 4.1$ & 0.829 \\
\hline \multirow[t]{3}{*}{ Diabetic retinopathy type } & Moderate NPDR + focal DME & $40(51.94 \%)$ & 25 (49.01\%) & \multirow[t]{3}{*}{0.801} \\
\hline & Severe NPDR+ diffused DME & $24(31.16 \%)$ & $15(29.41 \%)$ & \\
\hline & PDR+ diffused DME & $13(16.88 \%)$ & II (2I.56\%) & \\
\hline
\end{tabular}

Notes: Data were presented as mean \pm SD. Normally distributed variables of two groups were compared by unpaired Student's $t$-test. Not-normally distributed variables of the two groups were compared by the Mann-Whitney U-test. Categorical variables in two groups were presented as percentages (\%) and compared by the Chi-Square test. The study showed no statistically significant difference in baseline demographic parameters. A value of $p<0.05$ was considered statistically significant.

Abbreviations: RBZ, ranibizumab; BVZ, bevacizumab; DM, diabetes mellitus; HbAIc, glycated hemoglobin; NPDR, nonproliferative diabetic retinopathy; DME, diabetic macular edema; BCVA, best-corrected visual acuity; CMT, central macular thickness; IOP, intraocular pressure; SD, standard deviation.

The study also showed no statistically significant difference in the improvement of BCVA and reduction of CMT between two different anti-VEGF therapy groups (Table 2). Additionally, the two groups did not show any significant differences in terms of adverse events that occurred during therapy (Table 3). Regarding biochemical parameters, the study showed no significant differences in ROS generation of PBMC, serum level of AGE, and MDA among study groups (Table 4). The study showed no significant difference in the distribution of subjects with and without macular edema between RBZ+Laser therapy and BVZ+Laser therapy group after the end of 12 months (Table 5). The study also highlights the cost-effectiveness of both $\mathrm{RBZ}+$ laser and $\mathrm{BVZ}+$ laser therapies for the treatment of DME in DR. The results demonstrated that a subject

Table 2 Improvement in "Best Corrected Visual Acuity" (BCVA) and Decrease of Central Macular Thickness (CMT) at Month I2

\begin{tabular}{|c|c|c|c|}
\hline $\begin{array}{l}\text { Improvement in BCVA (in Terms of Letter Gain) and Decrease in CMT }(\mu \mathrm{m}) \text { from } \\
\text { Baseline to Month } 12\end{array}$ & $\begin{array}{l}\text { RBZ + } \\
\text { Laser }\end{array}$ & $\begin{array}{l}\text { BVZ + } \\
\text { Laser }\end{array}$ & p-value \\
\hline Mean change in BCVA in RBZ+Laser, $(n=77)$ and BVZ+Laser $(n=51)$ group & $6.87 \pm 5.53$ & $6.82 \pm 5.76$ & 0.858 \\
\hline $\begin{array}{l}\text { Mean change in BCVA among moderate NPDR+Focal DME subjects (RBZ+Laser, } n=40 \text {; BVZ+Laser, } \\
n=25)\end{array}$ & $11.30 \pm 3.603$ & $11.96 \pm 3.020$ & 0.540 \\
\hline $\begin{array}{l}\text { Mean change in BCVA among severe NPDR+Diffused DME subjects (RBZ+Laser, } n=24 ; B V Z+\text { Laser, } \\
n=15)\end{array}$ & $3.125 \pm 2.232$ & $3.200 \pm 2.455$ & 0.801 \\
\hline Mean change in BCVA among PDR+Diffused DME subjects $(R B Z+$ Laser, $n=24 ; B V Z+$ Laser, $n=15)$ & $0.153 \pm 0.3755$ & $0.090 \pm 0.301$ & 0.999 \\
\hline Mean change in CMT $(\mu \mathrm{m})$ in RBZ+Laser, $(n=77)$ and BVZ+Laser $(n=5 \mathrm{I})$ group & $140.1 \pm 38.00$ & $134.9 \pm 40.32$ & 0.300 \\
\hline $\begin{array}{l}\text { Mean change in CMT }(\mu \mathrm{m}) \text { among moderate NPDR+Focal DME subjects (RBZ+Laser, } n=40 ; B V Z \\
+ \text { Laser, } n=25)\end{array}$ & $171.9 \pm 3.770$ & $170.6 \pm 3.830$ & 0.192 \\
\hline $\begin{array}{l}\text { Mean change in CMT }(\mu \mathrm{m}) \text { among severe NPDR+Diffused DME subjects (RBZ+Laser, } n=24 \text {; BVZ } \\
+ \text { Laser, } n=15)\end{array}$ & $122.3 \pm 7.097$ & $|20| \pm 7.869$. & 0.375 \\
\hline $\begin{array}{l}\text { Mean change in CMT }(\mu \mathrm{m}) \text { among severe PDR+Diffused DME subjects (RBZ+Laser, } \mathrm{n}=24 \text {; BVZ+Laser, } \\
\mathrm{n}=15)\end{array}$ & $75.08 \pm 20.54$ & $74.18 \pm 20.84$ & 0.9168 \\
\hline
\end{tabular}

Notes: Data were presented as mean \pm SD. Normally distributed variables of two groups were compared by unpaired Student's $t$-test. Not-normally distributed variables of the two groups were compared by the Mann-Whitney U-test. No significant differences were observed in the recovery of VA and decrement of CMT when compared RBZ + Laser group with $B V Z+$ Laser groups. A value of $p<0.05$ was considered statistically significant.

Abbreviations: BCVA, best-corrected visual acuity; CMT, central macular thickness. 
Table 3 Showing the Adverse Events Related to Intravitreal Ranibizumab (RBZ) and Bevacizumab (BVZ)

\begin{tabular}{|c|c|c|c|}
\hline Adverse Events & $\begin{array}{c}\text { RBZ + } \\
\text { Laser } \\
(n=77)\end{array}$ & $\begin{array}{c}\text { BVZ + } \\
\text { Laser } \\
(n=5 I)\end{array}$ & p-value \\
\hline Arterial thromboembolic events & 0 & 0 & \multirow[t]{12}{*}{ - } \\
\hline Angina pectoris & I (1.29\%) & I (1.90\%) & \\
\hline Pulmonary embolism & 0 & 0 & \\
\hline Cerebrovascular accident & 0 & 0 & \\
\hline Myocardial infarction & 0 & 0 & \\
\hline Hypertension & $2(2.59 \%)$ & I (1.90\%) & \\
\hline Non-ocular hemorrhage & 0 & 0 & \\
\hline Vitreous hemorrhage & 0 & I (1.90) & \\
\hline Retinal detachment & 0 & 0 & \\
\hline Endophthalmitis & 0 & 0 & \\
\hline Raised IOP & 0 & $I(1.90)$ & \\
\hline Ocular pain & $3(3.89 \%)$ & $3(5.88 \%)$ & \\
\hline $\begin{array}{l}\text { Total number of subjects with } \\
\text { different kinds of adverse event }\end{array}$ & $6(7.79 \%)$ & 7 (I3.72\%) & \multirow[t]{2}{*}{0.276} \\
\hline $\begin{array}{l}\text { Number of subjects with no } \\
\text { adverse events }\end{array}$ & 71 (92.20\%) & 44 (86.27\%) & \\
\hline
\end{tabular}

Notes: Categorical variables in two groups were presented as percentages (\%) and compared by the Chi-Square test. In the RBZ + Laser treated group $7.79 \%$ of subjects (I.29\% angina pectoris, $2.59 \%$ hypertension, and $3.89 \%$ ocular pain) were found to be associated with different types of adverse events. In the BVZ + Laser treated group, $13.72 \%$ of subjects ( $1.90 \%$ angina pectoris, $1.90 \%$ hypertension, $1.90 \%$ vitreous hemorrhage, IOP raised among $1.90 \%$ and $5.88 \%$ subject experienced ocular pain) were found to be associated with different types of the adverse event. The rest $92 \%$ in RBZ + Laser treated group and $86.27 \%$ subjects in BVZ + Laser treated group experienced no adverse events. Subjects with adverse events and subjects without adverse events showed no significant distributional difference $(p=0.276)$ between the groups. A value of $p<0.05$ was considered statistically significant.

Abbreviation: IOP, intraocular pressure.

has to pay 20.951 times more cost (in INR) for RBZ +Laser therapy compared to BVZ+Laser therapy, to get an almost similar outcome (Table 6).

\section{Discussion}

It was evidenced from the different clinical trials that laser therapy alone is not effective for improved visual acuity recovery in subjects; rather, it is responsible for further vision loss among some patients of DR. ${ }^{14}$ The recent clinical trials demonstrated the efficacy and safety of intravitreal injections of RBZ for the treatment of DME. $^{15-17}$

Declining incidence of blindness related to ARMD and DME, coinciding with the advent of the anti-VEGF
Table 4 Levels of Different Biochemical Parameters After I 2 Months

\begin{tabular}{|l|l|l|l|}
\hline Parameters & $\begin{array}{l}\text { RBZ+Laser } \\
\text { Treated } \\
\text { Group }\end{array}$ & $\begin{array}{l}\text { BVZ+Laser } \\
\text { Treated } \\
\text { Group }\end{array}$ & p-value \\
\hline HbAIC (\%) & $7.97 \pm 2.92$ & $8.14 \pm 2.77$ & 0.7426 \\
\hline $\begin{array}{l}\text { PBMC ROS (Geo } \\
\text { mean of DCF/ 10 } \\
\text { cells) }\end{array}$ & $100.7 \pm 10.63$ & $98.54 \pm 16.28$ & 0.5112 \\
\hline $\begin{array}{l}\text { Serum AGE Conc. } \\
(\mu g / m L)\end{array}$ & $3.146 \pm 0.66$ & $3.02 \pm 0.803$ & 0.3068 \\
\hline $\begin{array}{l}\text { Serum MDA Conc. } \\
(\mathrm{nmol} / \mathrm{mL})\end{array}$ & $2.736 \pm 0.4948$ & $2.649 \pm 0.6435$ & 0.7293 \\
\hline
\end{tabular}

Notes: Data were presented as mean \pm SD. Not-normally distributed variables of two groups were compared by unpaired Mann Whitney U-test. The study showed no significant differences in $\mathrm{HbAlc}$, PBMC ROS, AGE, and MDA between study groups. A value of $p<0.05$ was considered statistically significant.

Abbreviations: PBMC, peripheral blood mononuclear cells; ROS, reactive oxygen species; DCF, Dichlorodihydrofluorescein Diacetate; AGE, advanced glycation end product; MDA, malondialdehyde.

Table 5 Distribution of Subjects with and without Clinically Significant Diabetic Macular Edema (CSDME) After 12 Months of Different Treatment Groups

\begin{tabular}{|l|c|c|c|}
\hline $\begin{array}{l}\text { Presence or } \\
\text { Absence of } \\
\text { CSDME }\end{array}$ & $\begin{array}{c}\text { RBZ+Laser } \\
\text { Treated } \\
\text { Group }\end{array}$ & $\begin{array}{c}\text { BVZ+Laser } \\
\text { Treated } \\
\text { Group }\end{array}$ & p-value \\
\cline { 1 - 3 } Presence & $28(36.36 \%)$ & $22(43.13 \%)$ & \multirow{2}{*}{0.464} \\
\cline { 1 - 3 } Absence & $49(63.63 \%)$ & $29(56.86 \%)$ & \\
\hline
\end{tabular}

Notes: Categorical variables in two groups were presented as percentages (\%) and compared by the Chi-Square test. In the RBZ + Laser treated group 28 (36.36\%) and 49 (63.63\%) subjects were found with CSME and without CSDME respectively after 12 months. In the BVZ+Laser treated group 22 (43.13\%) and 29 (56.86\%) subjects were found with CSDME and without CSDME respectively after 12 months. The statistical analysis showed no statistical difference in subjects distribution between the groups. A value of $\mathrm{P}<0.05$ was considered statistically significant.

therapy declared that anti-VEGF therapy had a clear superiority in efficacy and safety compared to laser therapy alone; however, the cost and frequent visits related to repeated intravitreal injections by clinicians create a substantial burden upon the health-care system and are a source of deep concern in our economic system. ${ }^{18,19}$

It has already been demonstrated that the usage of intravitreal RBZ therapy yields better results with other vision-threatening complications of DR, including PDR (proliferative DR) with DME. ${ }^{20}$ Diabetic macular edema, the most frequent complication of NPDR and PDR is caused by leakages from increased permeability or microaneurysms of retinal capillary beds and, given that it usually leads to vision loss due to macular edema, 
Table 6 Cost-Effective Regimen

\begin{tabular}{|l|c|c|c|c|c|c|c|c|}
\hline $\begin{array}{l}\text { Number } \\
\text { of Subjects } \\
\text { (RBZ + } \\
\text { Laser } \\
\text { Group) }\end{array}$ & $\begin{array}{c}\text { Number } \\
\text { of Subjects } \\
\text { BVZ + } \\
\text { Laser } \\
\text { Group }\end{array}$ & $\begin{array}{c}\text { Average } \\
\text { Letters } \\
\text { Gain } \\
\text { (RBZ + } \\
\text { Laser } \\
\text { Group) }\end{array}$ & $\begin{array}{c}\text { Average } \\
\text { Letters } \\
\text { Gain } \\
\text { (BVZ + } \\
\text { Laser } \\
\text { Group) }\end{array}$ & $\begin{array}{c}\text { Cost of } \\
\text { Treatment/ } \\
\text { Subject in } \\
\text { INR (RBZ + } \\
\text { Laser } \\
\text { Group) }\end{array}$ & $\begin{array}{c}\text { Cost of } \\
\text { Treatment/ } \\
\text { Subject in } \\
\text { INR (BVZ + } \\
\text { Laser } \\
\text { Group) }\end{array}$ & $\begin{array}{c}\text { Cost in INR/ } \\
\text { Letter Gain/ } \\
\text { Subject (RBZ } \\
\text { +Laser Group) }\end{array}$ & $\begin{array}{c}\text { Cost in INR / } \\
\text { Letter } \\
\text { Gain/Subject } \\
\text { (BVZ +Laser } \\
\text { Group) }\end{array}$ & $\begin{array}{c}\text { Cost } \\
\text { Increment } \\
\text { (Times in } \\
\text { INR)/Letter } \\
\text { Gain/ } \\
\text { Subject }\end{array}$ \\
\hline 77 & 51 & 6.87 & 6.823 & 52,500 & 1648.35 & 99.245 & 4.737 & 20.951 times \\
\hline
\end{tabular}

Notes: Cost-effectiveness was calculated in terms of cost increment (times in Indian national rate or INR)/letter gain/subject $=[$ Cost in INR/letter gain/ subject (RBZ +Laser group)]/[Cost in INR /letter gain/subject (BVZ + Laser group)]. The study showed that a subject has to pay 20.95I times more cost for a letter gain for RBZ + Laser therapy than the BVZ + Laser therapy.

mediated principally by VEGF, specifically angiogenic VEGF. $^{21,22}$ Different randomized clinical trials, DRCR. net, the RESTORE STUDY, and Protocol 1 compared the visual outcomes of patients having DME, with $0.5 \mathrm{mg}$ intravitreal RBZ with either prompt or deferred laser and demonstrated superior visual outcomes following this treatment modality in comparison to laser therapy alone. $^{15,23,24}$ The RESTORE study and Protocol $1^{15,24}$ suggested that adjunctive laser treatment does not appear to provide substantial visual benefits compared to ranibizumab treatment only, but may reduce the number of injections required to resolve DME. This protocol 1 also demonstrated similar visual outcomes following intravitreous triamcinolone plus prompt laser compared to intravitreous RBZ in pseudophakic patients but is associated with increased risk of raised intraocular pressure (IOP), need for glaucoma medications, and need for glaucoma surgery. Protocol T is the first trial to compare the efficacy and safety of the commercially available anti-VEGF drugs used in DME ie, RBZ, BVZ, and aflibercept. The top line DRCR.net protocol $\mathrm{T}$ results revealed improvement in vision from baseline to one year with all three drugs. Improvement was greater with aflibercept $(+13$ letters $)$ than RBZ $(+11$ letters $)$ or bevacizumab $(+10$ letters $){ }^{25}$ Reduction in retinal edema seen in patients treated with aflibercept was more compared to treatment with the other two drugs. DRCR.net Protocol 1 compared the significant cost difference between these agents-medicare allowable charges range from $\$ 1961$ for $2 \mathrm{mg}$ aflibercept, to $\$ 1189$ for $0.3 \mathrm{mg} \mathrm{RBZ}$ to $\$ 67$ for $1.25 \mathrm{mg} \mathrm{BVZ}$, but did not answer to any difference in efficacy and safety.

Alteration of pro- and antiangiogenic homeostasis of VEGF isoforms is now considered responsible for the severity of DR. ${ }^{26}$ Isoform-specific action of the antiVEGF agent is unknown till now.
Though an off-level drug, not approved by FDA, BVZ was given in India. It is a full-length humanized monoclonal antibody that binds to all types of VEGF, carries significant potentiality to block the VEGF-induced pathomechanism of DME. The BOLT trial showed a better margin of improvements far as BCVA is concerned-with $31 \%$ and $12 \%$ of patients achieving $\geq 10$ letters and $\geq 15$ letter gain, respectively - following intravitrealBVZ vis-àvis what was achieved following only focal/grid laser photocoagulation at 1 year namely, only $5 \%$ and $8 \%$ of the patients of DMEachieved comparable BCVA. ${ }^{27}$ A recent study compared three anti-VEGF agents, primarily, RBZ $0.5 \mathrm{mg}$, aflibercept $2.0 \mathrm{mg}$ and off-label BVZ $1.25 \mathrm{mg}$, for the treatment of center-involving DME, where the patients received an anti-VEGF drug at 4-week intervals for 24 weeks unless BCVA was 20/20 or better; and, which was followed by a retreatment algorithm involving monthly injection of the anti-VEGF agent, if worsening of BCVA; or, a focal/grid laser if there was no improvement in BCVA after 2 consecutive injections. This randomized trial demonstrated no significant differences in BCVA improvement at 1 year among the three anti-VEGF treatment groups, given the better baseline BCVA (20/32 - 20/40); whereas, aflibercept was associated with a significantly greater mean improvement in BCVA than the other two drugs among the patients of DME having the worse baseline BCVA (20/50 or less). ${ }^{28}$ The 2-year results of these three drugs declared almost similar vision improvement among patients with better presenting BCVA, whereas, aflibercept was associated with a greater mean BCVA improvement in patients with a worse presenting BCVA (20/50 or less). ${ }^{29}$

Present data demonstrated that the three intravitreal injections of RBZ along with laser therapy were effective for significant reduction of DME among 63.63\% of subjects with DME; whereas, the same improvement was seen in 
$56.86 \%$ of subjects who received off-label drugBVZ-pluslaser-photocoagulation. The study replicates the observations of the previous studies where anti-VEGF therapy-with-prompt -or-deferred-laser-photocoagulation results in greater VA gain vis-à-vis laser therapy alone. We also observed a similar trend of improvement in VA from a full-length monoclonal antibody ie, BVZ-plus-laser-photocoagulation. Further, the study did not reveal any significant difference in effectiveness for treatment outcome and safety measurement- for low-cost FDAunapproved full-length monoclonal antibody against VEGF compared to short-length anti-VEGF (RBZ), but both have shown promising results for moderate NPDR with focal DME. We also observed that the significant reduction of CMT in the RBZ-plus-laser-photocoagulation group $(140.1 \pm 38.0 \mu \mathrm{m})$ is not significantly higher than the significant reduction of the central macular thickness $(134.9 \pm 40.32 \mu \mathrm{m})$ in BVZ-plus-laser -therapy. Effectiveness and healthcare resource use in the 128 subjects - treated with anti-VEGF was assessed for antiVEGF-plus laser therapy for up to 1 year. The cost per subject in RBZ plus laser group was INR 52,500/- whereas the cost per subject under BVZ plus laser therapy was INR 1648.35/-. Cost for one letter improvement for a subject in RBZ therapy required INR 99.245/- whereas it was only INR 4.737/- in BVZ treatment (Table 6)

We believe our present observation might be crucial from the perspectives of the socio-economic well-being of our people as RBZ therapy is 20.951 times more expensive compared to its BVZ cohort. At the same time, no significant superior efficacy was observed for RBZ over BVZ that will encourage us who live in a little bit of a strain, under our financially constrained living standards scenario. It was further noticed that a huge population load, along with a health awareness shortcoming, seems to add additional hazards for India. So, the economic viability and sustainability of a low-cost offlabel anti-VEGF therapy, ie, BVZ, over the FDA-approved costly RBZ therapy will benefit the majority of the DRaffected Indian people. The study did not reveal any major complications related to either mode of therapy.

Though the painful laser therapy remained the gold standard for the treatment of $\mathrm{DR},{ }^{30}$ yet its precise and repeated use - along with its vision lowering complication cannot be provided to each individual due to the neglected ocular condition, at least in the Indian scenario where a huge population load along with awareness shortcomings create a deep concern for our developing country which now ranks second in the prevalence of DM along with its complication, DR. ${ }^{30}$ The three consecutive intravitreal injections of anti-VEGF drugs rapidly reduce DME and help regain visual acuity, where the additional effect of laser therapy in the less oedematous retina is the solution arriving naturally and easily, and that accumulates benefits for a longer period.

A recent study demonstrated different prognoses in patients with OCT-findings of hyper-reflective spots and serous retinal detachment with subretinal fluid treated with dexamethasone implant or ranibizumab injection. The structural OCT biomarkers including central retinal thickness, intraretinal cyst, choroidal thickness, serous detachment of neuroepithelium, and hyperreflective spot were applied to evaluate the effect of intravitreal RBZ and dexamethasone intravitreal implant on the treatment of DME and illustrated that DME associated with serous detachment of neuroepithelium and hyper-reflective spot represents a specific inflammatory pattern for which dexamethasone appears to be more effective. ${ }^{31}$ Recent animal and clinical studies strongly suggest a central inflammatory role in DME. Multiple cytokines and chemokines are involved in the pathogenesis of DME, with several cellular processes affecting the neurovascular unit. Specifically, serous detachment of neuroepithelium (SDN) and hyperreflective spots (HRS) have been recently proposed as non-invasive OCT imaging biomarkers of retinal inflammation in DME. ${ }^{32}$ A very recently, Meduri et al evaluated the efficacy and safety of intravitreal dexamethasone $0.7 \mathrm{mg}$ implant in treatment-naïve DME patients and assessed the utility of OCT structural biomarkers as predictors of functional response after treatment. Their study empathized the importance of structural biomarkers as predictors of favorable response and confirmed the efficacy and safety of intravitreal dexamethasone implant in treatmentnaïve DME patients showing a better functional response in the presence of serous retinal detachment (SRD), the integrity of ellipsoid zone (EZ) and absence of vitreomacular alterations. ${ }^{33}$ However, the structural damage of the retinal capillary wall occurs due to toxic effects of metabolites, mainly, anomalous glucose metabolism, lipid peroxidation, and endothelial cell injury- secondary to inflammatory leukostasis owing to the resultant response of increased secretion of VEGF. The hike in VEGF follows the consequent appearance and progression of DR. ${ }^{10}$ This study demonstrated the efficacy and cost-effectiveness of anti-VEGF agents aided by laser therapy to treat DME in the Indian population. Though the small sample size in a retrospective study design, the data derived from this study may shower some light on real-life experience on treatment of increasing blindness due to DME in developing and underdeveloped countries. 


\section{Conclusion}

In conclusion, the present study demonstrates the effectiveness of anti-VEGF therapy under different economic conditions, where BVZ is found to be the more attractive option for treating DME in DR for its cost-friendliness over RBZ in terms of BCVA outcome, as well as the safety perspectives, at least for the economically backward population in developing countries, like India. A modified treatment regimen consisting of the initial three monthly injections of BVZ or RBZ combined with focal/grid and/or scatter laser photocoagulation, may be a strong weapon to fight against the vision-impairment condition of patients with DME, especially in a developing country.

\section{Ethics and Consent}

The study was approved by the Institutional Ethics Committee (Medical College, Kolkata, Ref. No: MC/ KOL/IEC/NON-SPON/181/12-2018), and informed consents were collected from all patients according to the declaration of Helsinki.

\section{Acknowledgment}

Prof Ashim Kumar Ghosh, Director Regional Institute of Ophthalmology, for his encouragement and support towards the fulfillment of this work. UG and CB want to acknowledge CSIR, India, and DST, India for their fellowship.

\section{Funding}

This research did not receive any specific grant from funding agencies in the public, commercial, or not-forprofit sectors.

\section{Disclosure}

The authors report no conflicts of interest for this work.

\section{References}

1. Maguire MG, Martin DF, Ying GS, et al. Five-year outcomes with anti-vascular endothelial growth factor treatment of neovascular agerelated macular degeneration: the comparison of age-related macular degeneration treatments trials. Ophthalmology. 2016;123(8):17 51-1761. doi:10.1016/j.ophtha.2016.03.045

2. Kozak I, Shoughy SS, Stone DU. IntravitrealAntiangiogenic therapy of uveitic macular: a review. J Ocul Pharmacol Ther. 2017;33 (4):235-239. doi:10.1089/jop.2016.0118

3. Scott IU, VanVeldhuisen PC, Ip MS, et al. Effect of bevacizumab vs aflibercept on visual acuity among patients with macular due to central retinal vein occlusion: the SCORE2 Randomized Clinical Trial. J Am Med Assoc. 2017;317(20):2072-2087. doi:10.1001/jama.2017.4568
4. The Diabetic Retinopathy Clinical Research Network. Aflibercept, bevacizumab, or ranibizumab for diabetic macular Edema Two-year results from a comparative effectiveness Randomized Clinical Trial. N Engl J Med. 2015;372:1193-1203. doi:10.1056/ NEJMoa1414264

5. Payne JF, Wykoff CC, Clark WL, et al. Randomized trial of treat and extend ranibizumab with and without navigated laser for diabetic macular: TREX-DME1 year outcomes. Ophthalmology. 2017;124 (1):74-81. doi:10.1016/j.ophtha.2016.09.021

6. Jampol LM, Glassman AR, Bressler NM, et al. Anti-vascular endothelial growth factor comparative effectiveness trial for diabetic macular: additional Efficacy Post Hoc Analyses of a Randomized Clinical Trial. JAMA Ophthalmol. 2016;134(12):2016-3698. doi:10.1001/jamaophthalmol.2016.3698

7. World Health Organization. Global report on diabetes. Geneva: World Health Organization; 2016:1-88. Available from: https:// www.who.int/publications/i/item/9789241565257.

8. Yau JWY, Rogers SL, Kawasaki R, et al. Global prevalence and major risk factors of diabetic retinopathy. Diabetes Care. 2012;35 (3):556-564. doi:10.2337/dc11-1909

9. Elman MJ, Bressler NM, Qin H, et al. Expanded 2-year follow-up of ranibizumab plus prompt or deferred laser or triamcinolone plus prompt laser for diabetic macular. Ophthalmology. 2011;118 (4):609-614. doi:10.1016/j.ophtha.2010.12.033

10. Mondal LK, Bhaduri G, Bhattacharya B. Biochemical scenario behind initiation of diabetic retinopathy in type 2 diabetes mellitus. Indian J Ophthalmol. 2018;66(4):535-540. doi:10.4103/ijo.IJO 112117

11. Choudhuri S, Dutta D, Sen A, et al. Role of N-epsilon-carboxy methyl lysine, advanced glycation end products and reactive oxygen species for the development of nonproliferative and proliferative retinopathy in type 2 diabetes mellitus. Mol Vis. 2013; 19:100-113.

12. Satoh K. Serum lipid peroxide in cerebrovascular disorders determined by new colorimetric method. Clin Chim Acta. 1978;90:37-43. doi:10.1016/0009-8981(78)90081-5

13. Pramanik S, Ganguly U, Khemka VK, Banerjee A. Decreased glucose-6-phosphate dehydrogenase activity along with oxidative stress affects visual contrast sensitivity in alcoholics. Alcohol. 2018;73:17-24. doi:10.1016/j.alcohol.2018.03.007

14. Virgili G, Parravano M, Menchini F, et al. Anti-vascular endothelial growth factor for diabetic macular oedema. Cochrane Database Syst Rev. 2014;24(10):1-113.

15. Mitchell P, Bandello F, Schmidt-Erfurth U, et al. The RESTORE study: ranibizumab monotherapy or combined with laser versus laser monotherapy for diabetic macular. Ophthalmology. 2011;118 (4):615-625. doi:10.1016/j.ophtha.2011.01.031

16. Nguyen QD, Brown DM, Marcus DM, et al. Ranibizumab for diabetic macular: results from 2 phase 111 randomized trials: RISE and RIDE. Ophthalmology. 2012;119(4):789-801. doi:10.1016/j.ophtha. 2011.12.039

17. Elman MJ, Ayala A, Bressler NM, et al. IntravitrealRanibizumab for diabetic macular with prompt versus deferred laser treatment: 5-year randomized trial results. Ophthalmology. 2015;122(2):375-381. doi:10.1016/j.ophtha.2014.08.047

18. Azad R, Chandra P, Gupta R. The economic implications of use of anti-vascular endothelial growth factor drugs in age-related macular degeneration. Indian $J$ Ophthalmol. 2007;55(6):441-443. doi:10.4103/0301-4738.36479

19. Shanmugam PM. Changing paradigms of anti-VEGF in the Indian Scenario. Indian J Ophthalmol. 2014;62(1):88-92. doi:10.4103/03014738.126189

20. Gross JG, Glassman AR, Jampol LM, et al.; Writing Committee for the Diabetic Retinopathy Clinical Research Network. Panretinal photocoagulation vs intravitreous ranibizumab for proliferative diabetic retinopathy: a Randomized Clinical Trial. JAMA. 2015;314 (20):2137-2146. doi:10.1001/jama.2015.15217 
21. Moss SE, Klein R, Klein BE. The 14-year incidence of visual loss in a diabetic population. Ophthalmology. 1998;105(6):998-1003. doi:10.1016/S0161-6420(98)96025-0

22. Antonetti DA, Barber AJ, Hollinger LA, et al. Vascular endothelial growth factor induces rapid phosphorylation of tight junction proteins occludin and zonula occluden 1. A potential mechanism for vascular permeability in diabetic retinopathy and tumors. J Biol Chem. 1999;274(33):23463-23467. doi:10.1074/jbc.274. 33.23463

23. Elman MJ, Aiello LP, Beck RW, et al.; The Diabetic Retinopathy Clinical Research Network. Randomized trial evaluating ranibizumab plus prompt or deferred laser or triamcinolone plus prompt laser for diabetic macular edema. Ophthalmology. 2010;117(6):1064-1077. e35. doi:10.1016/j.ophtha.2010.02.031

24. Mukhamala L, Bhagat N, Zarbin MA. Practical lessons from Protocol 1 for the management of diabetic macular edema. Dev Ophthalmol. 2017;60:91-108.

25. Heier JS, Bressler NM, Avery RL, et al.; American Society of Retina Specialists Anti-VEGF for Diabetic Macular Edema comparative effectiveness panel. Comparison of afliberceft, bevacizumab, and ranibizumab for treatment of diabetic macular edema: extrapolation of data to clinical practice. JAMA Ophthalmol. 2016;134(1):95-99. doi:10.1001/jamaophthalmol.2015.4110

26. Paine SK, Mondal LK, Borah PK, et al. Pro- and antiangiogenic VEGF and its receptor status for the severity of diabetic retinopathy. Mol Vis. 2017;23:356-363.

27. Michaelides M, Kaines A, Hamilton RD, et al. A prospective randomized trial of intravitreal bevacizumab or laser therapy in the management of diabetic macular (BOLT Study) Study 12 month data: report 2. Ophthalmology. 2010;117(6):1078-1086. doi:10.1016/j. ophtha.2010.03.045
28. Wells JA, Glassman AR, Ayala AR, et al.; Diabetic Retinopathy Clinical Research Network. Aflibercept, bevacizumab or ranibizumab for diabetic macular edema. $N$ Engl $J$ Med. 2015;372 (13):1193-1203.

29. Wells JA, Glassman AR, Ayala AR, et al. Aflibercept, bevacizumab, or ranibizumab for diabetic macular: two-year results from a Comparative Effectiveness Randomized Clinical Trial. Ophthalmology. 2016;123 (6):1351-1360. doi:10.1016/j.ophtha.2016.02.022

30. Early Treatment Diabetic Retinopathy Study Research Group. Photocoagulation for diabetic macular. Early Treatment Diabetic Retinopathy Study report number 1. Early Treatment Diabetic Retinopathy Study research group. Arch Ophthalmol. 1985;103 (12):1796-1806. doi:10.1001/archopht.1985.01050120030015

31. Ceravolo I, Oliverio GW, Alibrandi A, et al. The application of structural retinal biomarkers to evaluate the effect of intravitreal ranibizumab and dexamethasone intravitreal implant on treatment of diabetic macular edema. Diagnostics. 2020;10:413. doi:10.3390/diagnostics10060413

32. Vujosevic S, Torresin T, Berton M, et al. Diabetic macular edema with and without subfoveal neuroretinal detachment: two different morphologic and functional entities. $\mathrm{Am} J$ Ophthalmol. 2017;181:149-155. doi:10.1016/j.ajo.2017.06.026

33. Meduri A, Oliverio GW, Trombetta L, et al. Optical coherence tomography predictors of favourable functional response in naïve diabetic macular edema eyes treated with dexamethasone implants as first-line agent. J Ophthalmol. 2021;2021:1-5. doi:10.1155/2021/6639418
Clinical Ophthalmology

\section{Publish your work in this journal}

Clinical Ophthalmology is an international, peer-reviewed journal covering all subspecialties within ophthalmology. Key topics include: Optometry; Visual science; Pharmacology and drug therapy in eye diseases; Basic Sciences; Primary and Secondary eye care; Patient Safety and Quality of Care Improvements. This journal is indexed on PubMed

\section{Dovepress}

Central and CAS, and is the official journal of The Society of Clinical Ophthalmology (SCO). The manuscript management system is completely online and includes a very quick and fair peer-review system, which is all easy to use. Visit http://www.dovepress.com/ testimonials.php to read real quotes from published authors. 